\title{
Effects of Neospora caninum Infection at Mid-Gestation on Placenta in a Pregnant Mouse Model
}

\author{
I. C. López-Pérez, E. Collantes-Fernández, S. Rojo-Montejo, V. Navarro-Lozano, V. Risco-Castillo, V. Pérez-Pérez*, J. Pereira-Bueno*, and \\ L. M. Ortega-Mora +, Saluvet, Animal Health Department, Faculty of Veterinary Sciences, Complutense University of Madrid, 28040 Madrid, Spain; \\ *Animal Health Department, Faculty of Veterinary Sciences, University of León, 24071 León, Spain.; †To whom correspondence should be \\ addressed.e-mail: luis.ortega@vet.ucm.es
}

\begin{abstract}
Neospora caninum is one of the more-efficient transplacentally-transmitted organisms. The goal of the present study was to investigate the pathologic and immunologic changes that occur at the materno-fetal interphase in pregnant BALB/c mice infected with $N$. caninum at mid-gestation. Parasite DNA was detected in feto-placentary units 3 days post-infection (PI). On day 7 PI, the DNA detection level and parasite burden were significantly higher in the placentas than in the fetuses, which may indicate that the parasite is mainly multiplying in the placenta during the initial infection. In the spleens of infected dams, we observed an increase in IFN- $\gamma$, IL-10, and IL-4. However, only IL-4 was upregulated in placentas from the infected dams; this may enhance susceptibility to $N$. caninum at the materno-fetal interphase and favor transmission to the progeny. Finally, an increase in TNF- $\alpha$ expression in nested-PCR-positive placentas combined with necrosis may compromise the viability of the fetuses.
\end{abstract}

Neospora caninum is one of the more-efficient transplacentallytransmitted organisms, and vertical transmission is responsible for the spread of infection from an infected dam to her offspring during pregnancy (Dubey et al., 2006). The timing of placental and fetal infections during gestation is an important factor in determining the disease outcome in both mice (Long and Baszler, 1996; Quinn et al., 2002; López-Pérez et al., 2006) and in experimental bovine infections (Williams et al., 2000; Innes et al., 2001). In a previous study, we observed the successful vertical transmission of $N$. caninum infection in BALB/c mice during 3 gestation periods. We demonstrated that infection at midgestation had the most severe consequences for the pups when compared to infection in early or late gestation, and leads to the highest rate of vertical transmission, fetal mortality, stillbirth, and a decrease in litter size (López-Pérez et al., 2006, 2008). Therefore, the aim of this study was to investigate the pathologic and immunologic changes that occur at the materno-fetal interphase in pregnant BALB/c mice infected with $N$. caninum on day 7 of gestation.

Eight-week-old female BALB/c mice (Harlan Interfauna Ibérica, Barcelona, Spain) were mated for 1 night, and day 0 of gestation was determined by the presence of the vaginal mucoid plug (López-Pérez et al., 2006, 2008). Pregnant BALB/c mice were infected subcutaneously with 2 $\times 10^{6} \mathrm{Nc}-1$ tachyzoites on day 7 of gestation. A group of pregnant mice was inoculated with PBS on day 7 of gestation and kept as an uninfected control group. Eight infected dams and 4 control animals were killed, at random, with $\mathrm{CO}_{2}$ gas at each time point. On day 10 of gestation (3 days PI), the complete feto-placentary unit was collected, while on day 14 of gestation (7 days PI), the fetuses, resorptions, placentas, and maternal spleens were recovered. The fetal mortality (resorptions) was determined on day 14 of gestation on the basis of small fetal size $(<3 \mathrm{~mm})$ or a lack of discernible fetal tissue at an implant site containing placenta tissue (Long and Baszler, 1996). The percent of resorption per dam was calculated as $\mathrm{R} /$ $(\mathrm{R}+\mathrm{V}) \times 100$ in every dam, where $\mathrm{R}$ is the number of resorbing fetuses and $\mathrm{V}$ is the number of viable fetuses per animal (Krishnan et al., 1996). The feto-placentary units, fetuses, and resorptions were individually processed for PCR analysis. All of the collected placentas were divided in 2 portions: one-half was always assayed using PCR analysis and the other half was processed for either cytokine expression or histological analysis. Finally, the spleens from dams were collected for cytokine expression analysis.

DOI: $10.1645 /$ GE-2347.1
A nested PCR was performed, as previously described, to detect parasite DNA in the tissue samples (Buxton et al., 1998; López- Pérez et al., 2006). In nested-PCR-positive samples, the $N$. caninum load was quantified by real-time PCR (Collantes-Fernández et al., 2002). Cytokine expression was evaluated by real-time RT-PCR. Total RNA was obtained by the commercial product TRI Reagent (Sigma, St. Louis, Missouri), and synthesis of cDNA was performed with SuperScript II Reverse Transcriptase (Invitrogen, Paisley, United Kingdom). The primer sequences for cDNA amplification of interferon $\gamma$ (IFN- $\gamma$ ), interleukin 10 (IL-10), interleukin 4 (IL-4), and $\beta$-actin have been previously published (Varona et al., 2005). The primer sequences for tumor necrosis factor $\alpha($ TNF- $\alpha)$ gene were as follows: forward "CTGTAGCCCACGTCGTAGC" and reverse "TTGAGATCCATGCCGTTG" (R. Varona, pers. comm.). The relative $\mathrm{n}$-fold changes of each target cytokine expression $(-\Delta \Delta \mathrm{Ct})$ were normalized to the endogenous reference $(\beta-$ actin) and related to the control group (Livak and Schmittgen, 2001). Routine histological methods were performed on formalin-fixed and paraffin-embedded placenta tissue sections. The analysis was based on the observation of lesions characteristic of, or consistent with, $N$. caninum infection (Long and Baszler, 1996). Local immune response and histological analyses were performed only in placentas containing live fetuses because, due to their small fetal size, resorptions were only recovered for parasite DNA detection.

The chi-square and Fisher $F$ tests were used to compare fetal mortality and the rates of parasite detection. The Mann-Whitney $U$-test was employed to analyze differences in the percent of resorption among infected and uninfected dams. The differences in the parasite burden were analyzed by a non-parametric Kruskal-Wallis test. When statistical differences were found, a Dunn's multiple comparison test was employed to examine all possible pairwise comparisons. The Student's $t$-test was used to compare the differences in cytokine expression between infected and uninfected dams. All of the statistical analyses were performed using GraphPad Prism v.5.02 software (San Diego, California).

The infected dams showed no body weight changes when compared to the control group (data not shown), and no clinical signs that were compatible with neosporosis were observed. The consequences of infection on the outcome of gestation were evaluated by investigating the fetal mortality in infected animals compared to uninfected dams (Table I). On day 14 of gestation, resorptions were observed in 6 of 8 infected dams and in 3 of 4 uninfected dams. We found an increase in fetal mortality $(P=$ $\left.0.671, \chi^{2}=0.18\right)$, and a higher percent of resorption per mouse $(P=$ $0.255, U=6.00$, Mann-Whitney $U$-test), in the infected animals compared to uninfected dams, although no significant differences were found. To analyze the pattern of parasite transmission from dams to fetuses when infection occurred at mid-gestation, the presence of $N$. caninum DNA (Table II) and parasite load (Fig. 1) were evaluated in the placentas and feto-placentary units or fetuses. Neospora caninum DNA was detected in feto-placentary units at 3 days PI (day 10 of gestation) and in the placentas and fetuses at 7 days PI (day 14 of gestation), with all of the dams showing at least 1 positive placenta. On day 14 of gestation, there was a significantly higher $N$. caninum detection $\left(P<0.0001, \chi^{2}=31.22, \chi^{2}\right.$ test $)$ and parasite load $\left(P<0.0001, \chi^{2}=68.98\right.$, Dunn's multiple comparison test) in the placentas compared to the fetuses. No differences in the parasite presence $(P=1$, Fisher $F$-test $)$ and parasite load $(P>0.05$, $\chi^{2}=68.98$, Dunn's multiple comparison test) were observed among the viable fetuses and resorptions. Finally, parasite DNA was more-often detected $\left(P<0.0001, \chi^{2}=38.12\right)$, and a higher parasite burden was observed ( $P<0.0001, \chi^{2}=68.98$, Dunn's multiple comparison test), in the placentas compared to the feto-placentary units. No parasite DNA 
TABLE I. Effect on the fetal mortality of Neospora caninum infection in dams infected on day 7 of gestation and sacrificed on day 14 of gestation (7 days $\mathrm{PI}$ )

\begin{tabular}{ccc}
\hline Group & \multicolumn{2}{c}{ Fetal mortality } \\
\hline Infected dams & $12 / 75(16.0 \%)^{*}$ & $20.0(4.6-21.7) \dagger$ \\
Control dams & $3 / 37(8.1 \%)$ & $10.0(10.0-11.0)$ \\
\hline
\end{tabular}

* Number of resorptions/number total viable fetuses (percentage).

$\uparrow$ Median of the percentage of resorptions per litter (lower and upper quartiles)

was detected in feto-placentary units, placentas, or fetuses from control mice.

To investigate if the local immune response differed from the systemic response, and to ascertain whether the infection at mid-gestation was associated with immunological changes at the materno-fetal interphase, we also evaluated cytokine expression in the spleens and placentas on day 14 of gestation (Fig. 2). We found a significant increase in IFN- $\gamma$, IL-10, and IL-4 mRNA expression levels in spleens from the infected dams compared to uninfected animals $(P<0.01-0.0001, t=3.81-11.24)$. In fetal placentas, only IL-4 was modified after infection, and a significant increase in its expression was observed in the infected dams compared to uninfected animals $(P<0.001, t=7.32)$. In addition, we compared cytokine expression in the nested-PCR-positive placentas compared to the negative placentas from infected mice (Fig. 3). The data show significantly higher TNF- $\alpha$ expression levels in the positive placentas $(P=0.042, t=$ 2.12 ). Finally, histological examination revealed multifocal areas of necrosis in both maternal and fetal sides of the placenta and minimal non-suppurative infiltrates. Necrosis and inflammatory infiltration were found in 39\% (9/23) and 13\% (3/23) of placentas, respectively. No lesions were observed in placentas from the control group.

The placenta may play a key role in the pathogenesis of neosporosis; in addition to being the natural barrier through which the parasite must cross to reach the fetus, the placenta can exhibit mechanisms of defense. However, relatively little is known about the role of the placenta during $N$. caninum infection in mice. Our data have demonstrated the presence of parasites in feto-placentary units as early as 3 days PI (day 10 of gestation). Nevertheless, it is unknown if the parasite reached the maternofetal interphase before day 3 PI because no mice were killed before this time. On day 14 of gestation (7 days PI), N. caninum DNA was detected in both the placentas and the fetuses. We found $N$. caninum DNA in a higher number of the placentas than in the fetuses, and the parasite burden in the placentas was also significantly higher than in the fetuses. Furthermore, parasite DNA was more-often detected in the placenta compared to the feto-placentary units, where an increase in the parasite load was also observed. In previous reports, in mice infected at late mid-gestation (day 10 of gestation), $N$. caninum tachyzoites were identified by immunohistochemistry in the placenta and fetus on days 6-8 PI (Long and Baszler, 1996; Rettigner et al., 2004). In a guinea pig model of Toxoplasma gondii infection, the placental parasite load was also time-dependent, and a higher parasite burden was found in the placenta compared to the fetuses in the early stages of the infection (Flori et al., 2003). Taken together, these findings may indicate that the parasite is mainly multiplying in the placenta during the initial infection. Although the parasite was detected in

TABle II. Detection of $N$. caninum DNA by nested PCR in fetoplacentary units, fetuses, resorptions, and placentas from mice infected on day 7 of gestation with $2 \times 10^{6} \mathrm{Nc}-1$ tachyzoites and sacrificed on days 10 ( 3 days PI) and 14 of gestation (7 days PI).

\begin{tabular}{lcc}
\hline \multirow{2}{*}{ Sample collected } & \multicolumn{2}{c}{ Nested PCR } \\
\cline { 2 - 3 } & No. samples* & Per litter $\dagger$ \\
\hline Feto-placentary units & $4 / 71(5.6 \%)$ & $5.0(0.0-1.1)$ \\
Viable fetus & $4 / 63(6.3 \%)$ & $0.0(0.0-13.9)$ \\
Resorptions & $1 / 12(8.3 \%)$ & $0.0(0.0-0.0)$ \\
Placentas & $35 / 60(58.3 \%)$ & $50.0(44.4-83.0)$ \\
\hline
\end{tabular}

* Number of positive samples/total of samples analyzed (percentage)

$\uparrow$ Median of positive samples per litter (minimum and maximum values)

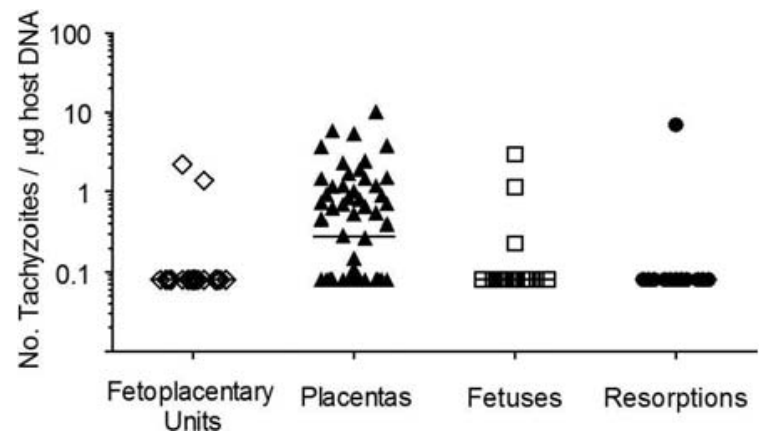

FIgURE 1. The parasite load in resorptions, placenta, fetuses, and fetoplacentary units following infection of dams with $2 \times 10^{6} \mathrm{Nc}-1$ tachyzoites on day 7 of gestation. The data are represented as individual points for the parasite load in each sample, and they are horizontally spread out for ease of visualizing overlapping values. The horizontal lane corresponds to the mean value. Considering the fact that the $N$. caninum detection limit by real-time PCR is $10^{-1}$ parasites (Collantes-Fernández et al., 2002), all of the positive samples had $\geq 0.1$ parasites while the negative samples ( 0 parasites) are represented on the log scale as $<0.1$ parasites.

very few fetuses $(6 \%)$ at this stage, the infection of a higher number of fetuses is likely to occur later in gestation because of the high vertical transmission detected previously on days 7 (López-Pérez et al., 2006) and 60 post-partum (López-Pérez et al., 2008).

The maintenance of a healthy pregnancy is associated with the secretion of anti-inflammatory cytokines. We attempted to determine if parasite infection at mid-gestation disrupts the materno-fetal immunological balance. We detected a significant increase in IFN- $\gamma$, IL-10, and IL-4 mRNA expression in the spleens from infected dams compared to uninfected animals. Previous reports have also described an increase in both Th1 and Th2 cytokine expression in $N$. caninum infection (Khan et al., 1997; Eperon et al., 1999; Quinn et al., 2004; Kano et al., 2005). In supernatants from mouse spleen cells stimulated with $N$. caninum antigen, an increase in IFN- $\gamma$, IL-12, TNF- $\alpha$, and IL-10 production was observed (Quinn et al., 2004), while an increase in IFN- $\gamma$ and IL-4 was detected in the serum (Kano et al., 2005) of pregnant mice. However, a different pattern of cytokine expression was observed in the placenta, where only IL-4 expression was upregulated in placentas from infected dams. IL-4 has been related to disease susceptibility in $N$. caninum-infected mice (Long et al., 1998; Baszler et al., 1999) and may favor the multiplication of parasites at the materno-fetal interphase (Quinn et al., 2004; Innes et al., 2005), leading to the high vertical transmission that has been previously observed after infection of dams at mid-gestation (López-Pérez et al., 2006, 2008). In addition, the increase in IL-4 expression might reduce the harmful effects of an antiparasitic pro-inflammatory immune response, which has been reported to be potentially deleterious for pregnancy maintenance

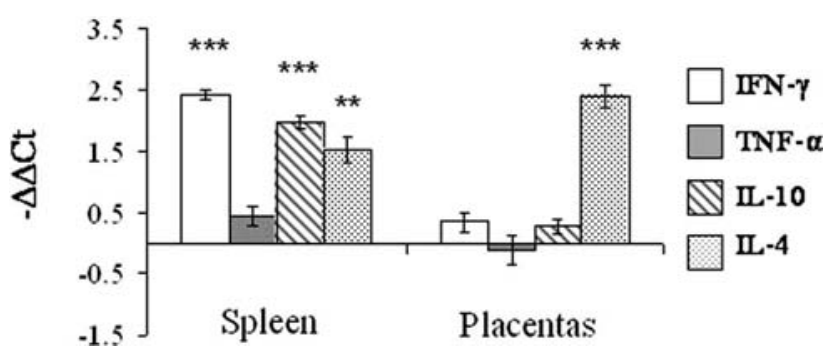

FIGURE 2. The cytokine expression in the spleen and fetal placentas from the infected dams compared to the uninfected animals. $\beta$-actin was used as an endogenous reference for each corresponding sample. Each determination was performed in triplicate, and the results of real-time RT$\mathrm{PCR}$ are given by $-\Delta \Delta \mathrm{Ct}$. The $-\Delta \Delta \mathrm{Ct}$ value for the control group is zero. The bars represent the mean of cytokine expression and the standard error. The asterisks represent the significant differences between the infected and uninfected dams using the Student's $t$-test $(* *: P<0.01$; ***: $P<0.001)$ 


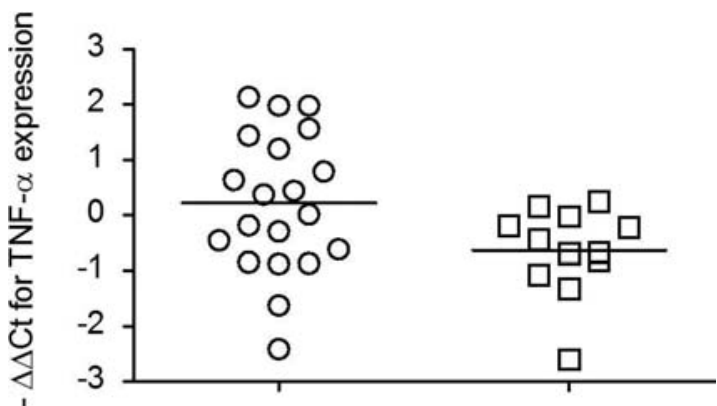

\section{Positive placentas Negative placentas}

FIGURE 3. TNF- $\alpha$ expression in the nested-PCR-positive and negative placentas from the infected dams. The data are represented as individual points in each sample, and the horizontal lane corresponds to the median value.

(Raghupathy, 1997). We found higher TNF- $\alpha$ mRNA levels in the nestedPCR-positive placentas, indicating that this cytokine may be produced in response to infection with $N$. caninum. TNF- $\alpha$ has been shown to be harmful to the maintenance of pregnancy in mice (Chaouat et al., 1990; Gazzinelli et al., 1996; Raghupathy, 2001), and given the high permeability of the blood-brain barrier during pregnancy, it may compromise the viability of the developing fetuses by causing brain damage (Michie, 1998). In addition, placental necrosis, which is likely to exacerbate as gestation progresses, could also be implicated in the high rates of stillbirth and neonatal mortality that have been previously observed (López-Pérez et al., 2006,2008 ) as a consequence of an insufficient supply of oxygen-nutrition by the placenta (Buxton et al., 1982).

A minimal, non-suppurative infiltrate was found in placentas from infected dams containing live fetuses. Previous reports have also demonstrated necrosis with few infiltrating inflammatory cells in placentas from pregnant mice infected on day 10 of gestation (Long et al., 1996). In $N$. caninum-infected cattle carrying live fetuses, and in pregnant control cattle, there was no evidence of infection of the placenta and, consequently, no inflammatory response (Maley et al., 2006). Altogether, these results suggest that the study of the inflammatory response in the placentas containing dead fetuses could more-precisely clarify the pathogenesis of $N$. caninum in pregnant mice.

In a previous study in cattle, a strong cytokine expression profile was observed in the placentomes of the cattle whose fetuses were killed as a result of infection (Rosbottom et al., 2008). However, we cannot discard the idea that a strong, type-1 immune response was responsible for the observed fetal resorptions because analysis of the local immune response was performed only in placentas containing live fetuses. Thus, further experiments are required to evaluate the importance of local and systemic immune response in fetal mortality.

In summary, this is the first report that describes the simultaneous analysis of parasite presence and load, the immune response, and the histopathological lesions in the placenta in BALB/c mice infected with $N$. caninum at mid-gestation.

This work has been supported by a grant from the Spanish Government (AGL2001-1362). Inmaculada C. López-Pérez has been financed by the Spanish Ministry of Education and Science. This experiment complied with the current local animal protection laws of the European Union.

\section{LITERATURE CITED}

Baszler, T. V., M. T. Long, T. F. McElwain, And B. A. Mathison. 1999. Interferon-gamma and interleukin-12 mediate protection to acute Neospora caninum infection in BALB/c mice. International Journal for Parasitology 29: 1635-1646.

Buxton, D., J. S. Gilmour, K. W. Angus, D. A. Blewett, and J. K. Miller. 1982. Perinatal changes in lambs infected with Toxoplasma gondii. Research in Veterinary Science 32: 170-176.

- S. W. Maley, S. Wright, K. M. Thomson, A. G. Rae, and E. A. INNES. 1998. The pathogenesis of experimental neosporosis in pregnant sheep. Journal of Comparative Pathology 118: 267-279.
Chaouat, G., E. Menu, D. A. Clark, M. Dy, M. Minkowski, and T. G. Wegmann. 1990. Control of fetal survival in CBA $\times$ DBA/2 mice by lymphokine therapy. Journal of Reproductive Fertility 89: $447-458$.

Collantes-Fernández, E., A. Zaballos, G. Álvarez-García, and L. M. Ortega-Mora. 2002. Quantitative detection of Neospora caninum in bovine aborted fetuses and experimentally infected mice by real-time PCR. Journal of Clinical Microbiology 40: 1194-1198.

Dubey, J. P., D. Buxton, And W. Wouda. 2006. Pathogenesis of bovine neosporosis. Journal of Comparative Pathology 134: 267-289.

Eperon, S., K. Brönnimann, A. Hemphill, and B. Gottstein. 1999. Susceptibility of B-cell deficient C57BL/6 $(\mu \mathrm{MT})$ mice to Neospora caninum infection. Parasite Immunology 21: 225-236.

Flori, P., J. Hafid, V. Thonier, B. Bellete, H. Raberin, and S. R. Tran MAnH. 2003. Parasite load in guinea pig foetus with real time PCR after maternofoetal transmission of Toxoplasma gondii. Parasite 10: 133-140.

Gazzinelli, R. T., M. Wysocka, S. Hieny, T. Scharton-Kersten, A. Cheever, R. Kuhn, W. Muller, G. Trinchieri, and A. Sher. 1996. In the absence of endogenous IL-10, mice acutely infected with Toxoplasma gondii succumb to a lethal immune response dependent on CD4+ T cells and accompanied by overproduction of IL-12, IFNgamma and TNF-alpha. Journal of Immunology 157: 798-805.

Innes, E. A., S. Wright, P. Bartley, S. Maley, C. Macaldowie, I. Esteban-Redondo, and D. Buxton. 2005. The host-parasite relationship in bovine neosporosis. Veterinary Immunology and Immunopathology 108: 29-36.

, S. Maley, A. Rae, A. Schock, E. Kirvar, P. Bartley, C. Hamilton, I. M. Carey, and D. Buxton. 2001. Protection against vertical transmission in bovine neosporosis. International Journal for Parasitology 31: 1523-1534.

Kano, R., Y. Masukata, Y. Omata, Y. Kobayashi, R. Maeda, and A SAITO. 2005. Relationship between type 1/type 2 immune responses and occurrence of vertical transmission in BALB/c mice infected with Neospora caninum. Veterinary Parasitology 129: 159-164.

Khan, I. A., J. D. Schwartzman, S. Fonseka, and L. H. Kasper. 1997. Neospora caninum: Role for immune cytokines in host immunity. Experimental Parasitology 85: 24-34.

Krishnan, L., L. J. Guilbert, T. G. Wegmann, M. Belosevic, and T. R. Mosmann. 1996. T helper 1 response against Leishmania major in pregnant $\mathrm{C} 57 \mathrm{BL} / 6$ mice increases implantation failure and fetal resorptions. Correlation with increased IFN-gamma and TNF and reduced IL-10 production by placental cells. Journal of Immunology 156: $653-662$.

Livak, K. And T. D. Schmittgen. 2001. Analysis of relative gene expression data using real-time quantitative PCR and the $2^{-\Delta \Delta C T}$ method. Methods 25: 402-408.

Long, M. T., AND T. V. BAszler. 1996. Fetal loss in BALB/c mice infected with Neospora caninum. Journal of Parasitology 82: 608-611.

, AND B. A. Mathison. 1998. Comparison of intracerebral parasite load, lesion development, and systemic cytokines in mouse strains infected with Neospora caninum. Journal of Parasitology 84: 316-320.

López-Pérez, I. C., E. Collantes-Fernández, A. Aguado-Martínez, A. Rodríguez-Bertos, and L. M. Ortega-Mora. 2008. Influence of Neospora caninum infection in BALB/c mice during pregnancy in post-natal development. Veterinary Parasitology 155: 175-183. , V. Risco-Castillo, E. Collantes-Fernández, and L. M. Ortega-Mora. 2006. Comparative effect of Neospora caninum infection in BALB/c mice at three different gestation periods. Journal of Parasitology 92: 1286-1291.

Maley, S. W. D. Buxton, C. N. Macaldowie, I. E. Anderson, S. E. Wright, P. M. Bartley, I. Esteban-Redondo, C. M. Hamilton, A. K. Storsety, And E. A. InNes. 2006. Characterization of the immune response in the placenta of cattle experimentally infected with Neospora caninum in early gestation. Journal of Comparative Pathology 135: 130-141.

Michie, C. 1998. Th1 and Th2 cytokines in pregnancy, from a fetal viewpoint. Immunology Today 19: 333-334.

Quinn, H. E., C. M. Miller, And J. T. Ellis. 2004. The cell-mediated immune response to Neospora caninum during pregnancy in the mouse is associated with a bias towards production of interleukin-4. International Journal for Parasitology 34: 723-732. 
C. Ryce, P. A. Windsor, and J. T. Ellis. 2002. Characterization of an outbred pregnant mouse model of Neospora caninum infection. Journal of Parasitology 88: 691696

Raghupathy, R. 1997. Th1-type immunity is incompatible with successful pregnancy. Immunology Today 18: 478-482.

. 2001. Pregnancy: Success and failure within the Th1/Th2/Th3 paradigm. Seminars in Immunology 13: 219-227.

Rettigner, C., F. De Meerschman, C. Focant, A. Vanderplasschen, AND B. Losson. 2004. The vertical transmission following the reactivation of a Neospora caninum chronic infection does not seem to be due to an alteration of the systemic immune response in pregnant CBA/Ca mice. Parasitology 128: 149-160.
Rosbottom, A., E. H. Gibney, C. S. Guy, A. Kipar, R. F. Smith, P. Kaiser, A. J. Trees, And D. J. Williams. 2008. An upregulation of cytokines is detected in the placenta of cattle infected with Neospora caninum, which is more marked early in gestation when foetal death is observed. Infection and Immunity 76: 2352-2361.

Varona, R., V. Cadenas, L. Gómez, A. Martinez, and G. Márquez. 2005. CCR6 regulates CD4+ T-cell-mediated acute graft-versus-host disease responses. Blood 106: 18-26.

Williams, D. J., C. S. Guy, J. W. McGarry, F. Guy, L. Tasker, R. F. Smith, K. MacEachern, P. J. Cripps, D. F. Kelly, and A. J. Trees. 2000. Neospora caninum-associated abortion in cattle: The time of experimentally-induced parasitaemia during gestation determines foetal survival. Parasitology 121: 347-358. 